\title{
Applications of diffusion-weighted imaging in diagnosis, evaluation, and treatment of acute ischemic stroke
}

\section{Oh Young Bang ${ }^{1}$, Wenyu $\mathrm{Li}^{2}$}

${ }^{1}$ Department of Neurology, Samsung Medical Center, Sungkyunkwan University School of Medicine, Seoul, Korea

${ }^{2}$ Department of Neurology, Sir Run Run Shaw Hospital, Medical School of Zhejiang University, Hangzhou, China

Received: March 18, 2019

Revised: May 10, 2019

Accepted: June 12, 2019

Corresponding author:

Oh Young Bang

Department of Neurology,

Samsung Medical Center,

Sungkyunkwan University

School of Medicine, 81 Irwon-

ro, Gangnam-gu, Seoul 06351 , Korea

Tel: +82-2-3410-3599

E-mail:

ohyoung.bang@samsung.com

\section{ABSTRACT}

Diffusion-weighted image (DWI) is widely used in acute care hospitals and provides diverse, valuable information about acute stroke. DWI is the gold standard for identifying the infarct core. The DWI infarct pattern is correlated with the pathogenic mechanisms underlying stroke and may predict stroke recurrence and outcome. Therefore, a deeper understanding of DWI is necessary for physicians treating patients with acute ischemic stroke. In this review, the application and interpretation of DWI for acute stroke and future perspectives are discussed.

Keywords: Cerebral infarction; Diffusion-weighted image; Magnetic resonance imaging; Stroke

\section{INTRODUCTION}

Magnetic resonance imaging (MRI) is a vital tool for the evaluation of acute stroke patients and has been used since the 1980s. The recent 2018 guidelines recommended that computed tomographic perfusion or diffusion-weighted image (DWI)/magnetic resonance (MR) perfusion scans should be obtained if the patient is past $>6$ hours after last known normal status and has large vessel occlusion [1].

Although various MR sequences have recently been developed and used due to their unique clinical value [2], DWI is widely used in acute care hospitals and provides diverse, valuable information about acute stroke. Therefore, a deeper understanding of DWI is necessary for physicians treating patients with acute ischemic stroke. In this review, the application and interpretation of DWI for acute stroke patients and perspectives are discussed.

This is an Open Access article distributed under the terms of the Creative Commons Attribution Non-Commercial License (http:// creativecommons.org/licenses/ by-nc/4.0/).

\section{DIFFUSION-WEIGHTED IMAGING}

DWI and apparent diffusion coefficient maps

DWI is the most reliable method for early detection of cerebral ischemia, definition of the in- 
farct core, and differentiation of acute ischemia from stroke mimics [3]. Within minutes of an ischemic insult, a core region of tissue exhibits profound loss of blood flow and becomes irreversibly damaged, even if blood flow is rapidly restored. DWI detects decreases in the self-diffusion of water molecules as early as 11 minutes after symptom onset [4]; these changes are probably related to cellular energy failure and early cytotoxic edema, reflecting the physiologic consequences of ischemic injury.

In general, the acquisition sequences for DWI generate b-1000 DWIs (typically utilized as the infarct core), b-0 DWIs, and apparent diffusion coefficient (ADC) maps. DWls should be reviewed with the ADC maps (diffusion-weighted without a T2 component) for proper interpretation. Combined with the ADC map, DWI provides information on the severity, reversibility, and chronology (age of infarcts) of ischemic brain damage. First, the $A D C$ is useful for estimating the lesion age and distinguishing acute from subacute DWI lesions [5]. Acute ischemic lesions can be divided into hyperacute (low $A D C$ and DWI-positive) and subacute (normalized ADC, 1 to 2 weeks after stroke onset) lesions, and chronic lesions can be differentiated from acute lesions by normalization of ADC and DWI. Second, tissues with a higher absolute ADC value have a higher likelihood of reversibility [6,7]. DWI lesions are commonly considered markers of irreversible ischemia, but can, occasionally, be reversed. DWI lesion reversal after reperfusion has been shown to be associated with early neurological improvement [8]. ADC threshold may be useful in distinguishing the ischemic core and penumbra, and the degree of reduction in $A D C$ values may predict $D W I$ lesion reversal after successful reperfusion [9]. However, the prevalence and clinical relevance of DWI reversal are unclear, although, a very low absolute $A D C$ value $\left(<550 \times 10^{-6} \mathrm{~mm}^{2} / \mathrm{sec}\right)$ and a large DWI lesion volume have been associated with severe, irreversible damage and a high risk of hemorrhagic transformation after thrombolysis $[10,11]$. Third, DWI with ADC maps can be useful in differentiation between vasogenic (elevated ADC) and cytotoxic (decreased ADC) edema [12]. Both areas of diminished and increased diffusion can appear bright on DWIs. In addition to arterial stroke, cerebral venous thrombosis can also cause stroke. Its main pathophysiological mechanisms are the breakdown of the blood-brain barrier, the coexistence of cytotoxic and vasogenic edema, and high prevalence of hemorrhage. Vasogenic edema can be identified as DWI-positive and elevated/normal ADC with peri-infarct swelling. In addition, gradient echo image may demonstrate susceptibility hypointensity within the thrombosed ve- nous sinus or cortical veins and hemorrhagic transformation.

\section{Diagnosis of stroke}

Diagnosis of stroke largely depends on clinical presentation. Stroke-mimics account for $19 \%$ to $30 \%$ of suspected stroke presentations, with diverse underlying etiology [13]. With advances in MRI technology, ischemic lesion can be identified with high accuracy using DWI (88\% to 100\% sensitivity, 95\% to $100 \%$ specificity). A false-negative DWI result is possible, and a meta-analysis including 3,236 acute ischemic stroke patients showed that the prevalence of DWI-negative acute ischemic stroke is $6.8 \%$ [14]. False-negative DWI was associated with less severe strokes, a longer time from onset to scan, small vessel disease, and localization in the posterior circulation, such as patients with small punctate infarcts located within the brainstem (e.g., as medulla) or deep gray nuclei (e.g., thalamus) [15]. In addition, 1.5-T DWI was better than 3.0-T DWI for imaging of hyperacute stroke within the first 6 hours [16]. False-positive DWI findings may occur in a variety of non-ischemic lesions, such as cerebral abscess (increased viscosity), lymphoma (dense cell packing), acute demyelination (myelin vacuolization), Creutzfeldt-Jakob disease (spongiform change), and encephalitis/seizure/transient global amnesia (cytotoxic edema) [3,17]. Most are readily distinguishable from acute infarcts if DWI findings are considered in conjunction with findings from other MRI sequences.

One-third of individuals with traditionally-defined transient ischemic attack (TIA) exhibit the signature of new infarction by DWI $[18,19]$, and application of DWI in TIA patients would reduce estimates of the annual incidence of TIA by $33 \%$ and increase estimates of the annual incidence of stroke in the United States by 7\% [20]. The percentage of TIA patients with a DWI lesion increases with increasing total symptom duration [21] and with increasing duration between TIA symptom onset and performance of the initial DWI [22]. TIA is now defined as a transient episode of neurological dysfunction caused by focal brain, spinal cord, or retinal ischemia without signs of acute infarction by neuroimaging [19]. Similarly, even in patients with transient neurological attacks, episodes of acute atypical or non-focal neurological symptoms, DWI showed acute ischemia in 23\% [23]. DWI lesions were associated with greater risk for stroke during the first 3 months after TIA [24]. Although the $A B C D^{2}$ score is widely used for TIA patients, the $A B C D^{3}-I$ score, which incorporates the presence of DWI lesions, has shown a higher predictive value for assessing the risk of early stroke after TIA $[25,26]$. These findings suggest that there is an important 
role for DWI in monitoring patients with transient symptoms, in both typical and atypical TIA.

\section{Stroke subtypes: assessment of the mechanisms of stroke}

DWI lesion patterns vary amongst patients with ischemic stroke. The DWI infarct pattern is correlated with the pathogenic mechanisms underlying the stroke and may predict stroke recurrence and outcome. Many studies have attempted to unravel stroke pathomechanisms using ischemic lesion topography by DWI because the size of clot at the site of occlusion, the characteristics of the clot (organized vs. fragile, red blood cell-rich vs. platelet-rich), and the proximity of clot (arteriogenic or aortocardiac) determine the DWI lesion pattern. In this context, DWI is valuable as a guide to specific diagnostic workups. For example, single cortical/subcortical lesions and multiple bilateral lesion in the anterior or posterior circulation, identified by DWI, have been associated with cardiac embolic sources, whereas multiple unilateral lesions in the anterior circulation have been linked to arteriogenic embolism $[27,28]$.

As clots from the left atrium or left atrial appendage are usually large (fibrin-containing, organized) and can occlude the distal internal carotid artery, proximal middle cerebral artery, or distal basilar artery, atrial fibrillation is associated with more severe ischemic stroke or longer (>60 minutes)
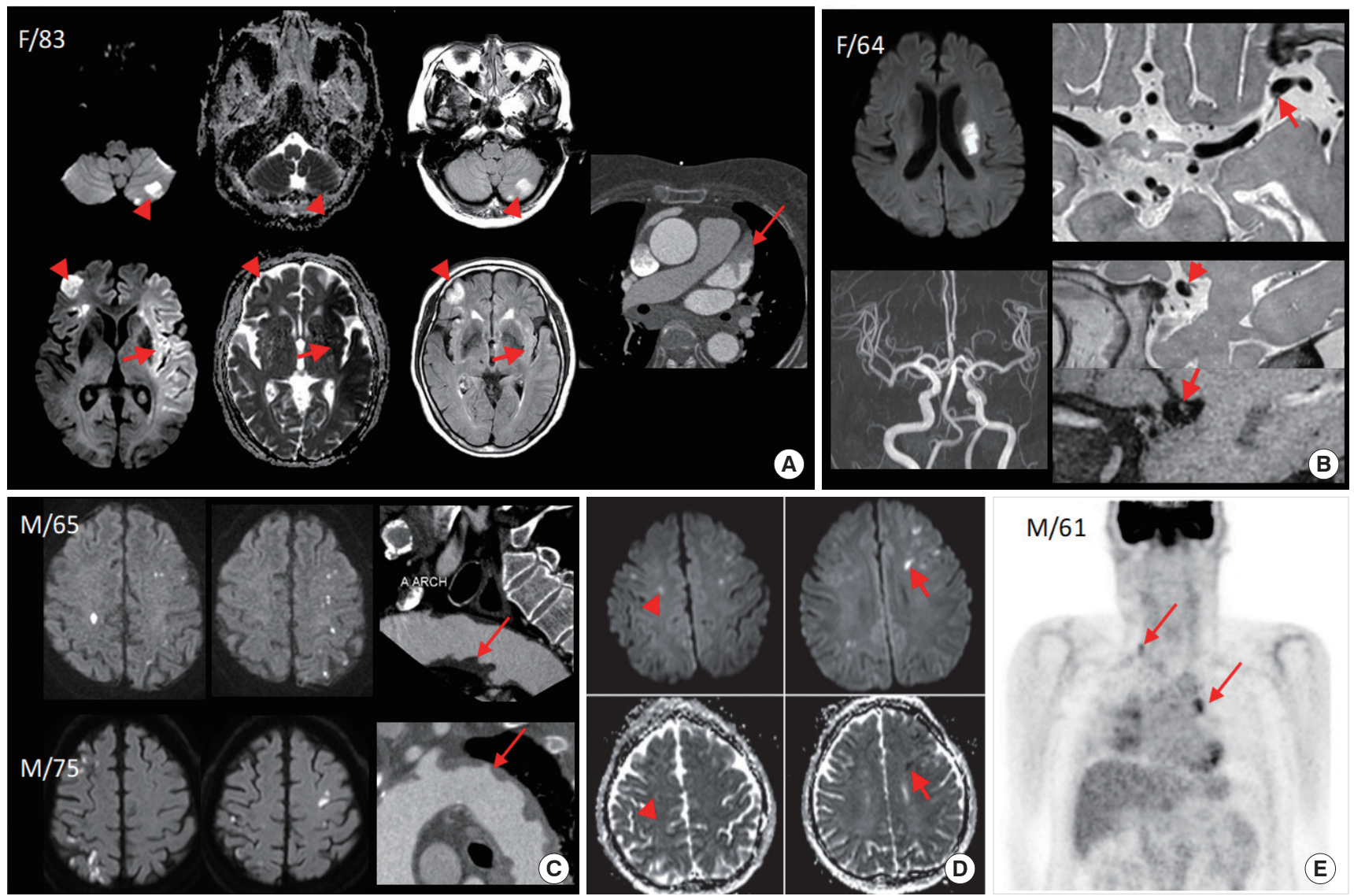

Fig. 1. Typical diffusion-weighted image (DWI) findings of various stroke etiopathologies. (A) A case with cardioembolic stroke due to atrial fibrillation. DWI shows large territorial infarcts involving the bilateral hemisphere and left cerebellum. The signal intensities of different magnetic resonance imaging (MRI) parameters vary after the onset of ischemic stroke, and some DWI lesions were hyperacute (low apparent diffusion coefficient [ADC] and fluid attenuated inversion recovery [FLAIR]-negative, arrows) and other DWI lesions were subacute (normal ADC and FLAIR-positive, arrowheads). Coronary computed tomography (CT) angiography showed thrombus in left atrial appendage (arrow). (B) A case with large, deep, comma-shaped infarcts, suggesting branch occlusive disease. Although no stenotic lesion was noticed on time-of-flight magnetic resonance angiography, high-resolution MRI revealed a small enhancing plaque at the origin of the perforators (arrows). (C) Two cases with aortic arch atheroma. DWI showed multiple, small, cortical border zone infarcts, bilaterally, and coronary CT angiography showed ulcerative atheroma of the aortic arch (arrows). (D) A case with cancer-related coagulopathy. DWI showed multiple lesions involving multiple territories, and co-existing subacute (arrowheads) and acute (arrows) infarcts. He presented with progressive, cognitive impairment but the work-up revealed no apparent explanation for stroke, and positron emission tomographic CT scan to screen for hidden malignancy revealed multiple lung cancer with metastasis. 
TIAs than arterio-embolic stroke from carotid or intracranial disease. Like cardiogenic embolism, both paradoxical embolism through the patent foramen ovale and aortogenic embolism cause multiple bilateral lesions or stroke recurrence in different vascular territory. However, they are both characterized by multiple, small, scattered lesions in multiple areas that are mainly located in cortical and border zone regions in aortic arch atheroma and in the cortical and posterior circulation in paradoxical embolism [29-31].

The topography of DWI lesions could be helpful in the application of diagnostic techniques, including cardiac telemonitoring, high-resolution wall imaging, and aortocardiac workups [32]. In an analysis of 321 patients with cryptogenic embolism, vascular risk factors, stroke severity, and DWI lesion patterns differ between those with paroxysmal atrial fibrillation, paradoxical embolism, and aortogenic embolism, indicating that patients with cryptogenic embolic stroke have distinct clinical and radiological features, depending on the underlying cause of stroke [33]. Fig. 1 shows typical DWI features of different stroke etiopathologies.

\section{DWI AND STROKE OUTCOMES}

\section{Stroke functional outcome: prediction of prognosis on admission}

DWI lesion volume determines the prognosis of patients with acute ischemic stroke, regardless of revascularization treatment/perfusion status [34]. There is emerging evidence that patients with a DWI lesion volume greater than approximately $70 \mathrm{~mL}$ do poorly [35]. Other than DWI lesion volume, the location of infarcts, as imaged by DWI, could influence functional outcome and stroke progression [36,37].

DWI lesion pattern may help in the recognition of differences in early prognostic endpoints after ischemic stroke, and may also guide targeted interventions to prevent negative outcomes (Fig. 2). In a prospective analysis of 426 patients

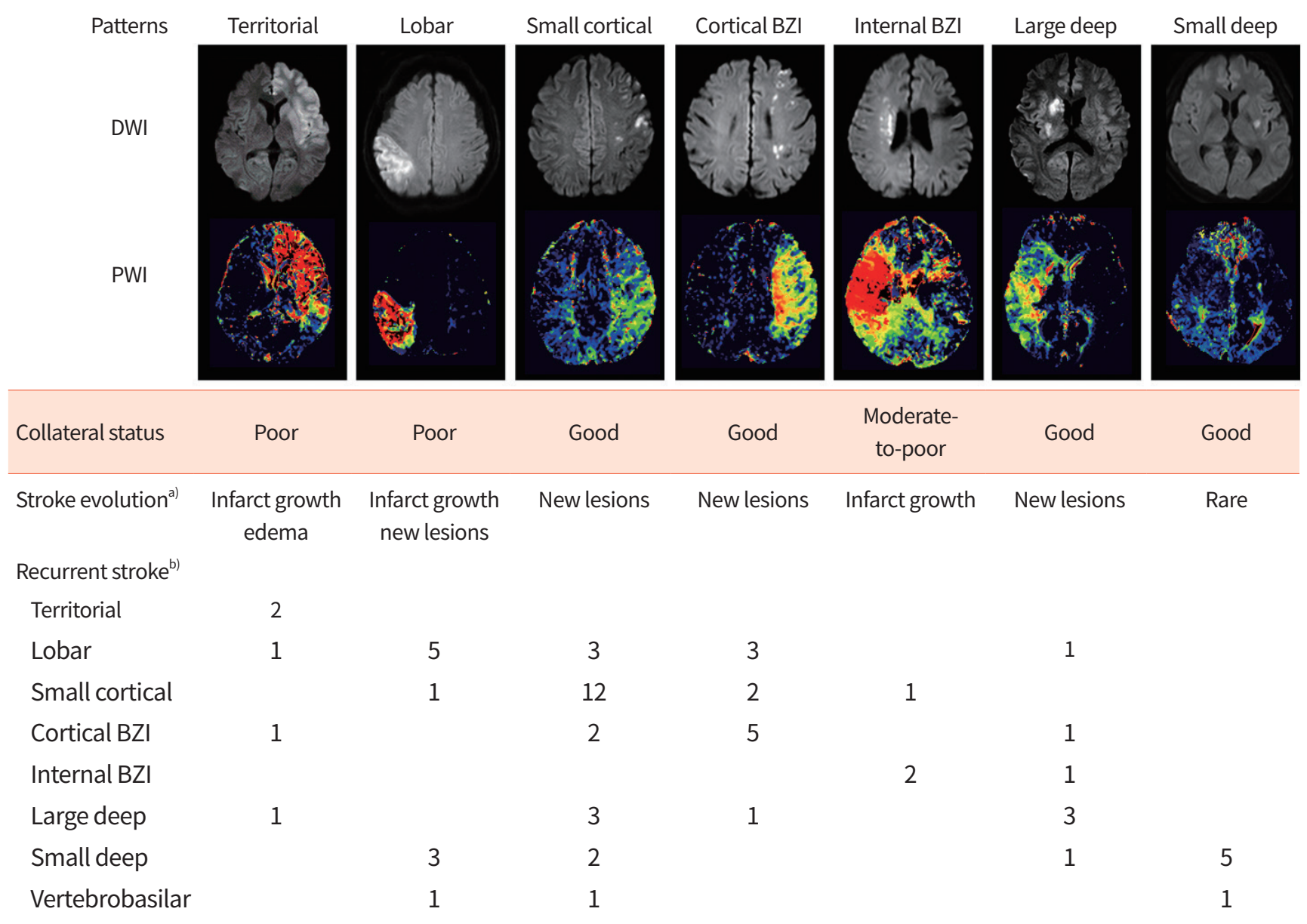

Fig. 2. Seven diffusion-weighted image (DWI) lesion patterns. Each DWI lesion pattern shows differential hemodynamic status (perfusionweighted image features and collateral status), early outcomes (stroke evolution), and late outcomes (DWI lesion pattern of recurrent strokes). BZI, border zone infarct; PWI, perfusion-weighted image. ${ }^{\text {a) }}$ Most frequent type; ${ }^{\text {b) }}$ Unpublished data. 
with acute infarcts in the middle cerebral artery territory, an unstable hospital course was frequently observed in patients with internal border zone infarcts, and poor outcome after stroke was associated with older age, severe neurological deficits at admission, and a DWI lesion pattern showing internal border zone infarcts [38]. Changes in DWI lesion volumes at follow-up (72 to 96 hours) were varied, but large, in internal border zone infarcts, compared with other DWI lesion patterns ( $226 \%$ vs. $143 \%$, compared with initial DWI lesion volume). Recurrent strokes after the index stroke were common in those who had small superficial infarcts [38]. The presence of multiple DWI lesions of varying ages is suggestive of active, early recurrences over time and portends a greater, early risk of future ischemic events [39].

These differences in stroke outcome, depending on DWI lesion pattern, could be resultant from the severity of perfusion deficits and collateral status. Stroke evolution frequently occurs during the first week after stroke. An analysis of the association between the type of stroke evolution and baseline perfusion severity showed that there was no association between two types of stroke evolution: 'new lesions' (new lesions not contiguous with initial DWI lesions) and 'infarct growth' (enlargement of infarct size) [40]. Most new lesions were multiple and small, located in cortical/superficial areas, and within areas of mild perfusion deficit, whereas infarct growth generally occurred within regions with more severe perfusion deficits, suggesting differential therapeutic approaches for these two types of stroke evolution. Most patients who showed infarct growth had a territory/lobar- or internal border zone-type DWI lesion pattern; while patients with new lesions showed small cortical or cortical border zone infarct patterns. Collateral status determines outcome after stroke [41], and it differs between DWI lesion patterns. Compared to territorial/lobar type DWI lesion patterns, deep or small superficial patterns are associated with good collateral status [42].

In addition, DWI lesion volume (and extent of perfusion deficits) determines the speed of clinical recovery after revascularization therapy. Not all patients show dramatic improvement after revascularization therapy, and a significant proportion of patients who lack early clinical improvement after days of successful revascularization still achieved good outcomes at 3 months (Stunned brain syndrome) [43]. In consecutive patients who underwent pretreatment DWI, a significant correlation was noted between the time to $>40 \%$ reduction in National Institutes of Health Stroke Scale score and initial DWI lesion volumes [44]. In this study, pretreat- ment DWI lesion volumes of $>13.8 \mathrm{~mL}$ forecast a high probability of absence of early, dramatic improvement within 24 hours after successful revascularization.

\section{Recurrent stroke pattern: prediction of subsequent stroke pattern at discharge}

We have performed a retrospective analysis of patients to test whether there is a correlation in DWI lesion pattern between index and recurrent stroke (unpublished data). A total of 65 patients with anterior circulation infarcts, confirmed by DWI, recurred during the follow-up period. The most frequent DWI pattern of the recurrent infarcts was that of the index stroke (34 of 65 patients, 52.3\%), suggesting that the DWI lesion pattern of the index stroke determined that of recurrent stroke (Fig. 2). Such findings could be caused by the fact that the recurrence rate and types of recurrence are greatly dependent on subtypes of the index stroke $[45,46]$. In a large, randomized trial of ischemic stroke patients, about half of recurrent stroke were caused by the same mechanisms as the index stroke [46].

\section{CONCLUSION}

In summary, DWI is the most widely used MRI technique and can provide are variety information about acute stroke. DWI is the gold standard for identifying infarct core. The infarct pattern as seen on DWI is correlated with the pathogenic mechanisms underlying the stroke and may predict stroke recurrence and outcome. Continuous research on DWI and other MR techniques will help achieve the goal of an individualized etiopathological approach for diagnostic workups of stroke mechanism and for tissue-based decision-making in acute stroke revascularization therapy.

With the advances in transformative technologies (such as machine learning and artificial intelligence), along with a better understanding of stroke pathophysiology and MRI physics, it is highly likely that the clinical use of DWI will increase in the near future. The main limitation of MRI is a longer scanning time compared to that of non-contrast computed tomography (CT). However, there have been advances in MRI scanning and automatic post-processing. Although a comprehensive MRI protocol can be implemented in about 20 minutes, a fast, multimodal MRI protocol can be implemented in about 6 minutes, rivaling the time of any comprehensive acute stroke CT protocol [47]. In addition, automatic detection and measurement of DWI values are increasingly being used in clinical trials. For example, the RApid postpro- 
PRECISION AND FUTURE MIEDICINE

Diffusion-weighted imaging in acute ischemic stroke

cess for Perfuslon and Diffusion (RAPID, Rapid Software Corp., Grapevine, TX, USA), an automated software package for performing quantitative evaluation of $A D C$ to estimate the ischemic core and perfusion-weighted image thresholding of $T_{\max }>6$ seconds for defining critical hypoperfusion, has been used in several randomized clinical trials (RCTs) [48]. Second, until now, no clinical trials involving acute stroke patients have used DWI as a surrogate endpoint. Very recently, incidence of new DWI lesions were used as the primary endpoint in a randomized trial comparing the effects of clopidogrel and ticlopidine/ginkgo in clopidogrel-resistant patients undergoing carotid artery stenting [49]. With advances in fast-scanning and automatic measurement of DWI values [2], DWI could be used as an inclusion endpoint and surrogate outcome endpoint in acute stroke RCTs. Third, application of artificial intelligence in the acute stroke field is increasing. It can be particularly helpful in decision-making in every step of revascularization therapy for acute ischemic stroke: in clinical and imaging recognition of acute ischemic stroke in the ambulance or emergency room and in predicting the outcome after endovascular therapy [50-52]. Lastly, a quantitative radiomics approach for a more detailed analysis of DWI features, such as signal intensity or lesion texture (e.g., surface area and sphericity), could be helpful in differentiation of stroke subtypes [53].

\section{CONFLICTS OF INTEREST}

No potential conflict of interest relevant to this article was reported.

\section{ACKNOWLEDGMENTS}

This research was supported by a grant from the Bio \& Medical Technology Development Program of the National Research Foundation (NRF) funded by the Korean government (MSIT) (2018M3A9H1023675).

\section{ORCID}

Oh Young Bang https://orcid.org/0000-0002-7962-8751

Wenyu Li https://orcid.org/0000-0002-3590-6801

\section{REFERENCES}

1. Powers WJ, Rabinstein AA, Ackerson T, Adeoye OM, Bambakidis NC, Becker K, et al. 2018 Guidelines for the early management of patients with acute ischemic stroke: a guideline for healthcare professionals from the American Heart Association/American Stroke Association. Stroke 2018;49:e46-110.

2. Bang OY, Chung JW, Son JP, Ryu WS, Kim DE, Seo WK, et al. Multimodal MRI-based triage for acute stroke therapy: challenges and progress. Front Neurol 2018;9:586.

3. Fung SH, Roccatagliata L, Gonzalez RG, Schaefer PW. MR diffusion imaging in ischemic stroke. Neuroimaging Clin N Am 2011;21:345-77.

4. Hjort N, Christensen S, Solling C, Ashkanian M, Wu O, Rohl $L$, et al. Ischemic injury detected by diffusion imaging 11 minutes after stroke. Ann Neurol 2005;58:462-5.

5. Lansberg MG, Thijs VN, O'Brien MW, Ali JO, de Crespigny AJ, Tong DC, et al. Evolution of apparent diffusion coefficient, diffusion-weighted, and T2-weighted signal intensity of acute stroke. AJNR Am J Neuroradiol 2001;22:63744.

6. Schlaug G, Benfield A, Baird AE, Siewert B, Lovblad KO, Parker RA, et al. The ischemic penumbra: operationally defined by diffusion and perfusion MRI. Neurology 1999; 53:1528-37.

7. Gonen KA, Simsek MM. Diffusion weighted imaging and estimation of prognosis using apparent diffusion coefficient measurements in ischemic stroke. Eur J Radiol 2010;76:157-61.

8. Labeyrie MA, Turc G, Hess A, Hervo P, Mas JL, Meder JF, et al. Diffusion lesion reversal after thrombolysis: a MR correlate of early neurological improvement. Stroke 2012; 43:2986-91.

9. Olivot JM, Mlynash M, Thijs VN, Purushotham A, Kemp S, Lansberg MG, et al. Relationships between cerebral perfusion and reversibility of acute diffusion lesions in DEFUSE: insights from RADAR. Stroke 2009;40:1692-7.

10. Singer OC, Humpich MC, Fiehler J, Albers GW, Lansberg MG, Kastrup A, et al. Risk for symptomatic intracerebral hemorrhage after thrombolysis assessed by diffusion-weighted magnetic resonance imaging. Ann Neurol 2008;63:52-60.

11. Selim M, Fink JN, Kumar S, Caplan LR, Horkan C, Chen Y, et al. Predictors of hemorrhagic transformation after intravenous recombinant tissue plasminogen activator: prognostic value of the initial apparent diffusion coefficient and diffusion-weighted lesion volume. Stroke 2002; 33:2047-52.

12. Chu K, Kang DW, Yoon BW, Roh JK. Diffusion-weighted magnetic resonance in cerebral venous thrombosis. Arch Neurol 2001;58:1569-76. 
13. Gibson LM, Whiteley W. The differential diagnosis of suspected stroke: a systematic review. J R Coll Physicians Edinb 2013;43:114-8.

14. Edlow BL, Hurwitz S, Edlow JA. Diagnosis of DWI-negative acute ischemic stroke: a meta-analysis. Neurology 2017;89:256-62.

15. Simonsen CZ, Madsen MH, Schmitz ML, Mikkelsen IK, Fisher M, Andersen G. Sensitivity of diffusion- and perfusion-weighted imaging for diagnosing acute ischemic stroke is $97.5 \%$. Stroke 2015;46:98-101.

16. Rosso C, Drier A, Lacroix D, Mutlu G, Pires C, Lehericy S, et al. Diffusion-weighted MRI in acute stroke within the first 6 hours: 1.5 or 3.0 Tesla? Neurology 2010;74:1946-53.

17. Dmytriw AA, Sawlani V, Shankar J. Diffusion-weighted imaging of the brain: beyond stroke. Can Assoc Radiol J 2017;68:131-46.

18. Brazzelli M, Chappell FM, Miranda H, Shuler K, Dennis M, Sandercock PA, et al. Diffusion-weighted imaging and diagnosis of transient ischemic attack. Ann Neurol 2014;75: 67-76.

19. Easton JD, Saver JL, Albers GW, Alberts MJ, Chaturvedi S, Feldmann E, et al. Definition and evaluation of transient ischemic attack: a scientific statement for healthcare professionals from the American Heart Association/ American Stroke Association Stroke Council; Council on Cardiovascular Surgery and Anesthesia; Council on Cardiovascular Radiology and Intervention; Council on Cardiovascular Nursing; and the Interdisciplinary Council on Peripheral Vascular Disease. The American Academy of Neurology affirms the value of this statement as an educational tool for neurologists. Stroke 2009;40:2276-93.

20. Ovbiagele B, Kidwell CS, Saver JL. Epidemiological impact in the United States of a tissue-based definition of transient ischemic attack. Stroke 2003;34:919-24.

21. Kidwell CS, Alger JR, Di Salle F, Starkman S, Villablanca P, Bentson J, et al. Diffusion MRI in patients with transient ischemic attacks. Stroke 1999;30:1174-80.

22. Shono K, Satomi J, Tada Y, Kanematsu Y, Yamamoto N, Izumi Y, et al. Optimal timing of diffusion-weighted imaging to avoid false-negative findings in patients with transient ischemic attack. Stroke 2017;48:1990-2.

23. van Rooij FG, Vermeer SE, Goraj BM, Koudstaal PJ, Rich-

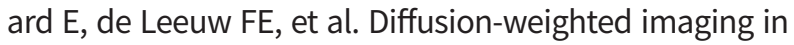
transient neurological attacks. Ann Neurol 2015;78:100510.

24. Calvet D, Touze E, Oppenheim C, Turc G, Meder JF, Mas JL. DWI lesions and TIA etiology improve the prediction of stroke after TIA. Stroke 2009;40:187-92.

25. Merwick A, Albers GW, Amarenco P, Arsava EM, Ay H, Calvet $D$, et al. Addition of brain and carotid imaging to the $A B C D(2)$ score to identify patients at early risk of stroke after transient ischaemic attack: a multicentre observational study. Lancet Neurol 2010;9:1060-9.

26. Song B, Fang H, Zhao L, Gao Y, Tan S, Lu J, et al. Validation of the ABCD3-I score to predict stroke risk after transient ischemic attack. Stroke 2013;44:1244-8.

27. Lee LJ, Kidwell CS, Alger J, Starkman S, Saver JL. Impact on stroke subtype diagnosis of early diffusion-weighted magnetic resonance imaging and magnetic resonance angiography. Stroke 2000;31:1081-9.

28. Wessels T, Wessels C, Ellsiepen A, Reuter I, Trittmacher S, Stolz E, et al. Contribution of diffusion-weighted imaging in determination of stroke etiology. AJNR Am J Neuroradiol 2006;27:35-9.

29. Kim SJ, Ryoo S, Hwang J, Noh HJ, Park JH, Choe YH, et al. Characterization of the infarct pattern caused by vulnerable aortic arch atheroma: DWI and multidetector row CT study. Cerebrovasc Dis 2012;33:549-57.

30. Kent DM, Ruthazer R, Weimar C, Mas JL, Serena J, Homma $\mathrm{S}$, et al. An index to identify stroke-related vs incidental patent foramen ovale in cryptogenic stroke. Neurology 2013;81:619-25.

31. Kim JW, Kim SJ, Yoon CW, Park CH, Kang KW, Kim SK, et al. Association between the amount of right-to-left shunt and infarct patterns in patients with cryptogenic embolic stroke: a transcranial Doppler study. Int J Stroke 2013;8: 657-62.

32. Bang OY, Ovbiagele B, Kim JS. Evaluation of cryptogenic stroke with advanced diagnostic techniques. Stroke 2014;45:1186-94.

33. Ryoo S, Chung JW, Lee MJ, Kim SJ, Lee JS, Kim GM, et al. An approach to working up cases of embolic stroke of undetermined source. J Am Heart Assoc 2016;5:e002975.

34. Kruetzelmann A, Kohrmann M, Sobesky J, Cheng B, Rosenkranz M, Rother J, et al. Pretreatment diffusion-weighted imaging lesion volume predicts favorable outcome after intravenous thrombolysis with tissue-type plasminogen activator in acute ischemic stroke. Stroke 2011;42:1251-4.

35. Yoo AJ, Barak ER, Copen WA, Kamalian S, Gharai LR, Pervez MA, et al. Combining acute diffusion-weighted imaging and mean transmit time lesion volumes with National Institutes of Health Stroke Scale score improves the prediction of acute stroke outcome. Stroke 2010;41:172835. 
PRECISION AND FUTURE MIEDICINE

Diffusion-weighted imaging in acute ischemic stroke

36. Cheng B, Forkert ND, Zavaglia M, Hilgetag CC, Golsari A, Siemonsen $\mathrm{S}$, et al. Influence of stroke infarct location on functional outcome measured by the modified rankin scale. Stroke 2014;45:1695-702.

37. Oh S, Bang OY, Chung CS, Lee KH, Chang WH, Kim GM. Topographic location of acute pontine infarction is associated with the development of progressive motor deficits. Stroke 2012;43:708-13.

38. Bang OY, Lee PH, Heo KG, Joo US, Yoon SR, Kim SY. Specific DWI lesion patterns predict prognosis after acute ischaemic stroke within the MCA territory. J Neurol Neurosurg Psychiatry 2005;76:1222-8.

39. Sylaja PN, Coutts SB, Subramaniam S, Hill MD, Eliasziw M, Demchuk AM, et al. Acute ischemic lesions of varying ages predict risk of ischemic events in stroke/TIA patients. Neurology 2007;68:415-9.

40. Bang OY, Kim GM, Chung CS, Kim SJ, Kim KH, Jeon P, et al. Differential pathophysiological mechanisms of stroke evolution between new lesions and lesion growth: perfusion-weighted imaging study. Cerebrovasc Dis 2010;29: 328-35.

41. Bang OY, Goyal M, Liebeskind DS. Collateral circulation in ischemic stroke: assessment tools and therapeutic strategies. Stroke 2015;46:3302-9.

42. Bang OY, Saver JL, Alger JR, Starkman S, Ovbiagele B, Liebeskind DS, et al. Determinants of the distribution and severity of hypoperfusion in patients with ischemic stroke. Neurology 2008;71:1804-11.

43. Alexandrov AV, Hall CE, Labiche LA, Wojner AW, Grotta JC. Ischemic stunning of the brain: early recanalization without immediate clinical improvement in acute ischemic stroke. Stroke 2004;35:449-52.

44. Bang OY, Liebeskind DS, Saver JL, Kim GM, Chung CS, Lee $\mathrm{KH}$, et al. Stunned brain syndrome: serial diffusion perfu- sion MRI of delayed recovery following revascularisation for acute ischaemic stroke. J Neurol Neurosurg Psychiatry 2011;82:27-32.

45. Shin DH, Lee PH, Bang OY. Mechanisms of recurrence in subtypes of ischemic stroke: a hospital-based follow-up study. Arch Neurol 2005;62:1232-7.

46. Toni D, Di Angelantonio E, Di Mascio MT, Vinisko R, Bath PM; PRoFESS Study Group. Types of stroke recurrence in patients with ischemic stroke: a substudy from the PRoFESS trial. Int J Stroke 2014;9:873-8.

47. Nael K, Khan R, Choudhary G, Meshksar A, Villablanca P, Tay J, et al. Six-minute magnetic resonance imaging protocol for evaluation of acute ischemic stroke: pushing the boundaries. Stroke 2014;45:1985-91.

48. Straka M, Albers GW, Bammer R. Real-time diffusion-perfusion mismatch analysis in acute stroke. J Magn Reson Imaging 2010;32:1024-37.

49. Chung JW, Kim SJ, Hwang J, Lee MJ, Lee J, Lee KY, et al. Comparison of clopidogrel and ticlopidine/ginkgo biloba in patients with clopidogrel resistance and carotid stenting. Front Neurol 2019;10:44.

50. Lee EJ, Kim YH, Kim N, Kang DW. Deep into the brain: artificial intelligence in stroke imaging. J Stroke 2017;19: 277-85.

51. Abedi V, Goyal N, Tsivgoulis G, Hosseinichimeh N, Hontecillas R, Bassaganya-Riera J, et al. Novel screening tool for stroke using artificial neural network. Stroke 2017;48: 1678-81.

52. Asadi H, Dowling R, Yan B, Mitchell P. Machine learning for outcome prediction of acute ischemic stroke post intra-arterial therapy. PLoS One 2014;9:e88225.

53. Cheng B, Knaack C, Forkert ND, Schnabel R, Gerloff C, Thomalla G. Stroke subtype classification by geometrical descriptors of lesion shape. PLoS One 2017;12:e0185063. 